\title{
UAV Mission Definition and Implementation for Visual Inspection
}

\author{
Tomáš Adam \\ Department of Cybernetics and \\ Artificial Intelligence, Faculty of \\ Electrical Engineering and \\ Informatics, Technical University \\ of Košice, Letná 9, 04001 Košice, \\ Slovakia \\ Email: tomas.adam@tuke.sk
}

\begin{abstract}
Although Unmanned Aerial Vehicles (UAVs) are currently still in a state of continuous development, the trend of their integration into a wide range of industries and applications is growing. It leads to risk and cost reduction but requires skilled operators. This fact motivated us to propose a new approach towards a UAV-based flight mission-definition system based on state-of-the-art waypoint-based techniques. Our solution enables the configuration and autonomous conduction of flight trajectories, whose spatial complexity exceeds the visualization capabilities of currently available solutions. Two testing scenarios confirmed our expectations; the autonomous execution of the trajectory reduced the time required in all cases by almost a half while achieving the same output as a user-controlled manual flight. The proposed solution extends the possibilities of users in creating complex flight trajectories and significantly contributes to the higher time efficiency of recurrent flights.
\end{abstract}

\section{INTRODUCTION}

$\mathrm{W}$ HILE the potential of the UAVs has been used in the past, mainly in the military sector, the continuing trend of increasing integration in the civil and commercial spheres has made it possible to increase the effectiveness of several activities in various fields. The potential use of these devices supports existing areas and helps to create new segments of application and development for commercial and civil purposes. The major benefit of UAVs excels mainly in the activities whose character requires, above all, unrestricted movement in space. This feature contributes to increasing the safety of the participating users and creates a tool that provides a view from different perspectives without having to overcome vertical obstacles.

\section{A. Related Work}

UAVs in agriculture bring several benefits, especially in the field of multi-spectral scanning and mapping. Vegetation indices of the infrared part of the light spectrum, for instance, the Normalized Difference Vegetation Index (NDVI) [1] can be used for the vegetation mapping, which can provide information about the vegetation stress, soil composition, fertilization quality, pest infestations, nutrient deficiencies, water stress, and other relevant conditions

\author{
František Babič \\ Department of Cybernetics and \\ Artificial Intelligence, Faculty of \\ Electrical Engineering and \\ Informatics, Technical University \\ of Košice, Letná 9, 04001 Košice, \\ Slovakia \\ Email: frantisek.babic@tuke.sk
}

affecting crop productivity. This approach makes the capturing of induced abiotic stress before the reactions begin to appear in the visible part of the light spectrum [2].

UAVs in the energy industry find application in many scenarios, but mainly in control and prevention activities or damage investigations after natural disasters. Using UAVs in solar power plants allows regular inspection and maintenance of photovoltaic panels placed on inclined structures and inaccessible places through thermo-graphic imaging and diagnostics. This approach is currently the cheapest and fastest way to maintain and identify hot spots (which are among the defects that may cause the most destructive effects) in large solar power plants without the need to install fixed sensors and cameras [3, 4].

Checking the condition of wind power plants with UAVs allows access to wind turbines and their blades, which can currently reach a length of more than a hundred meters without using costly elevating platforms, scaffoldings, and industrial climbing methods [5]. According to Ian Glenn, CEO of ING Robotic Avionics, inspecting a single wind turbine using UAVs is $50 \%$ cheaper and takes an average of 1.5 to 3 hours, which is 3 to 4 times more time-efficient than conventional methods'. This process's availability and efficiency help increase the life of entire wind farms and prevent damage that, if neglected, may necessitate the replacement of the entire wind turbine blade [6].

The transmission and distribution networks are complex system with many components whose failure can lead to serious problems. During power line inspections, UAVs have significant advantages compared to conventional human expert-based and helicopter-based methods. They allow automated and contactless inspection of electric power distribution systems in high resolution with minimal downtimes and interruptions in the power supply [7]. Unlimited movement in space makes it possible to achieve higher efficiency of the visual inspection, allowing a point of view of individual components from several angles that cannot be reached during conventional inspections [8]

1http://insideunmannedsystems.com/ing-robotic-aviation-usesresponder-uas-for-turbine-blade-inspections/ 
The paper is organized as follows: the introduction briefly presents the motivation for proposing the new solution for visual inspections using UAVs. The second chapter describes the solution's design, including the user interface for flying missions' definition and implementation. The experiments cover two scenarios demonstrating the benefits of the proposed solution; the final chapter concludes the paper.

\section{PROPOSED SOLUTION}

This section describes briefly the technological details of our solution. The architecture is composed of two primary subsystems (Fig. 1). The Ground Control Station (GCS) module and the Unmanned Aerial Vehicle (UAV).

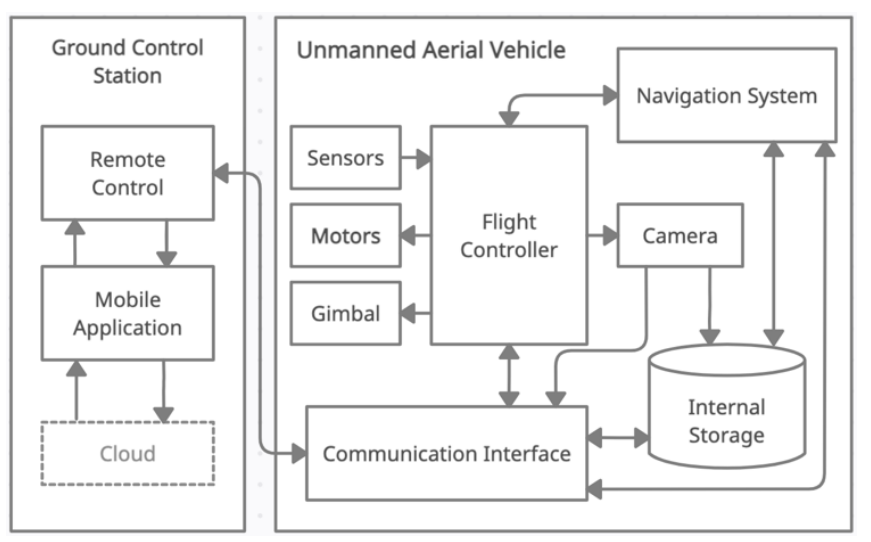

Fig. 1 Mission definition system

The UAV represents the quadrotor itself, which includes multiple cooperating parts: the flight controller (FC) performs mission execution and the flight based on the instructions from GCS. Then navigation system, helping with the orientation and correction of the actual UAV position with desired flight trajectory. The capture device (video, thermal camera, or lidar) performs the measurements and visual inspection. The captured data of these measurements should be stored in the internal SD card storage of the UAV with a time and GPS reference. The communication interface provides the primary wireless communication between UAV and GCS.

The Ground Control Station (GCS) is composed of several components. The remote controller represents the main component for wireless communication with the UAV, providing mainly user-based real-time flight instructions via joystick levers and buttons. It also provides communication access for the mobile application functions (as detailed in Section B) with a mission-definition interface and cloud storage services.

\section{A. Mission Visualization and Preparation}

The iOS mobile application represents the graphical user interface (GUI) for user interaction with specific functions, which exceeds the UAV remote controller capabilities. The primary function of this part aims at flight mission definition and its preparation for execution. The internal architecture depicted in Fig. 2 shows the flight mission specification conversion from data defined by the user to the mission flight plan used by the UAV and trajectory visualization in the GUI. These data may represent the previously saved flight mission, or the actual flight trajectory created by the user through GUI. The process can be decomposed into several phases.

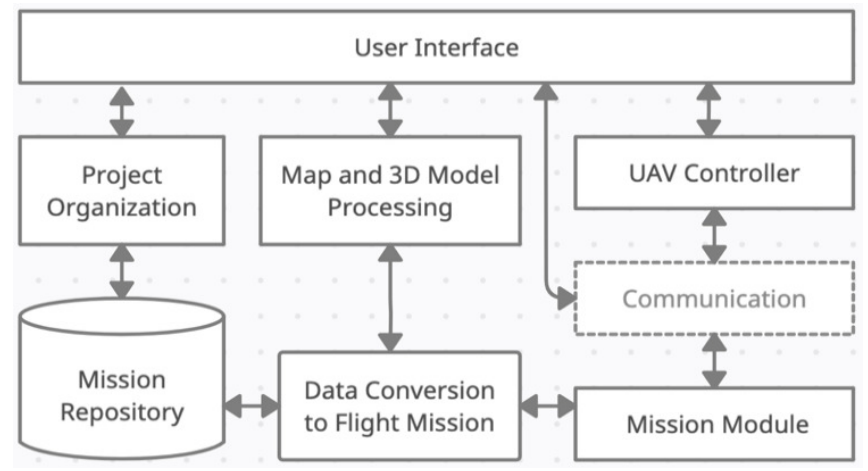

Fig. 2 Internal architecture

First, the mission specification data defined in the GUI is converted from the flight trajectory visualization module to a flight mission and saved into the database. Then the flight mission is validated and prepared for execution by the UAV. Finally, after validation, successful preflight control of the UAV peripherals, and loading the flight mission into its internal memory, the mission execution module starts the defined path execution.

\section{B. Mission Definition Interface}

The main layout depicted in Figure 3. comprises two main parts: mission definition and trajectory visualization. The mission definition part is represented by multiple sections containing saved flight missions, trajectory definition, and waypoint configuration. The parameters in this panel change according to the selected mission or waypoint with defined GPS coordinates, altitude, rotation, flight speed, and action.
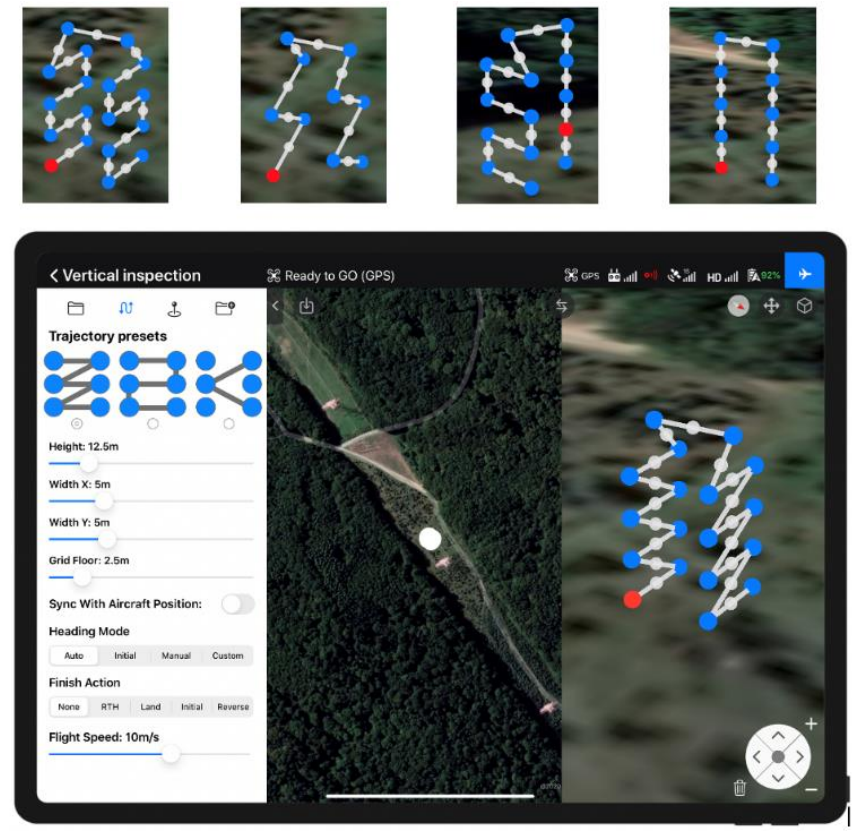

Fig. 3 Main interface of the mission definition tool 
The second part is represented by a $2 \mathrm{D}$ and $3 \mathrm{D}$ trajectory visualization to provide the required level of customization for the user, without limiting the ability to edit and display the complex flight trajectories.

\section{EXPERIMENTS AND RESULTS}

Two scenarios were chosen to demonstrate the benefits of the proposed solution and the increase of the UAV flight efficiency on basic flight trajectories.

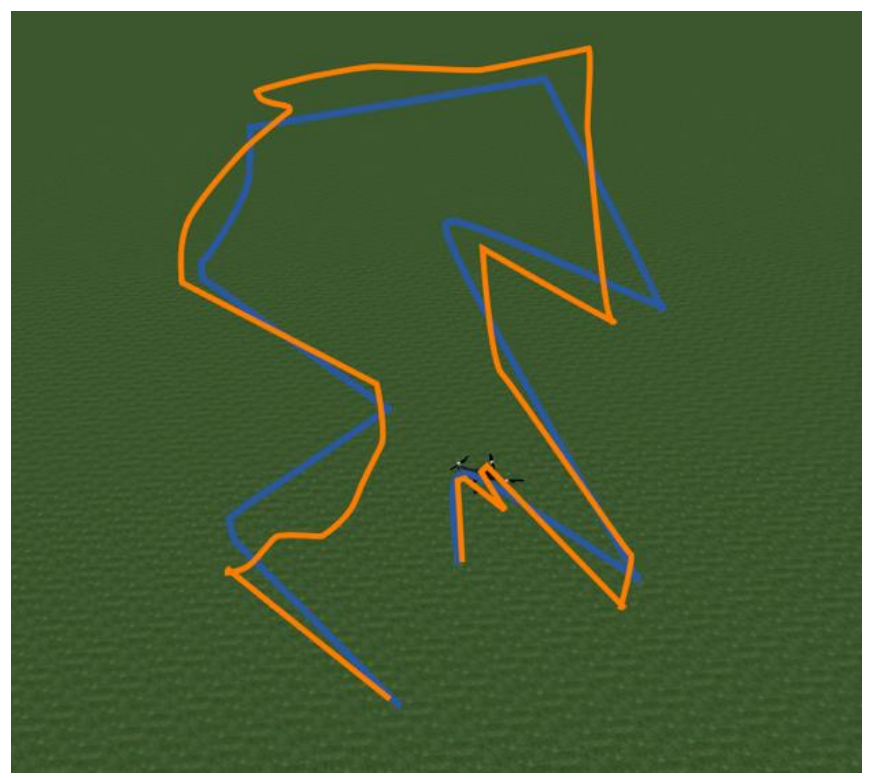

Fig. 4 Mission trajectory visualization

The first scenario illustrates the comparison of the autonomous flight of the UAV representing the ideal flight trajectory created through the mission definition interface, with the similar flight trajectory performed manually by the user. These trajectories are color-coded, where blue indicates an autonomous flight and orange a manual flight conducted by the user. The primary focus of these test flights was to compare GPS coordinates (Fig. 4, 5, and 6), the flight altitude, and the time required to complete the task.

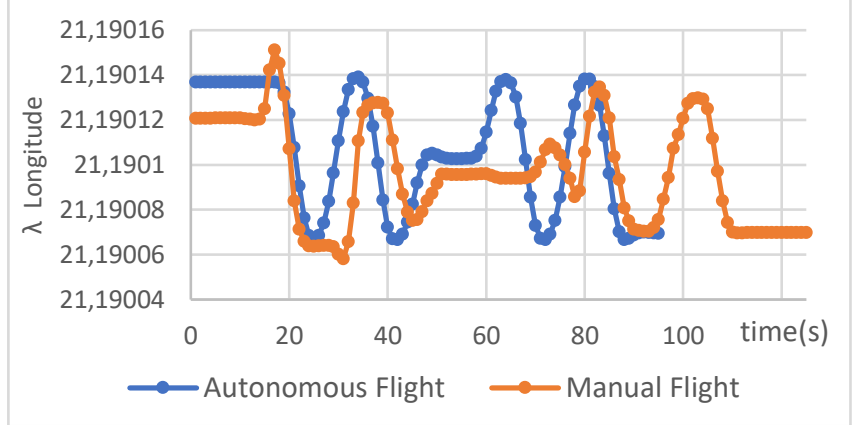

Fig. 5 Visualization of the first test: Longitude

For a more suitable display and simplification of recording deviations in the flight performance, a trajectory of $5 \times 5 \times 10$ meters was chosen with a northeast wind of $5 \mathrm{~m} / \mathrm{s}$ to increase the authenticity of the simulation. Ten flights were performed during the testing, from which the flight with the lowest deviation from the autonomously performed reference trajectory was selected (Fig. 4). During these flights, the trajectories were recorded and visualized through graphs showing the change in latitude and longitude values over time (Figs. 5 and 6).

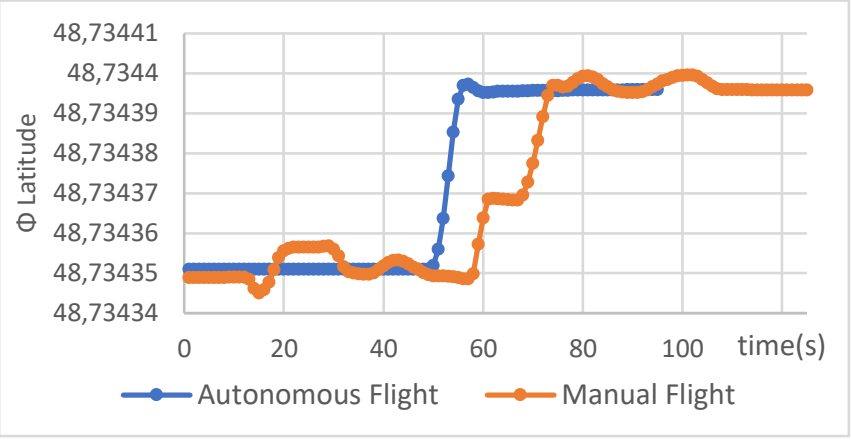

Fig. 6 Visualization of the first test: Latitude

Visualization of the overall course of the test (Fig. 5 and 6) shows that the manual execution of the trajectory took $35 \%$ longer and contained several errors caused mainly by the inertia of the UAV and the time required for rotation in the upper part of the flight trajectory.

The second scenario illustrates the time effectiveness of a repetition of the flight trajectory flown by the user manually. In this part, the main focus was on the ability of the proposed application to replicate the flight trajectory and the performed actions. The reference trajectory represents a simulation of the visual inspection of a large structure with the rotation of the device and the creation of images at different height levels.

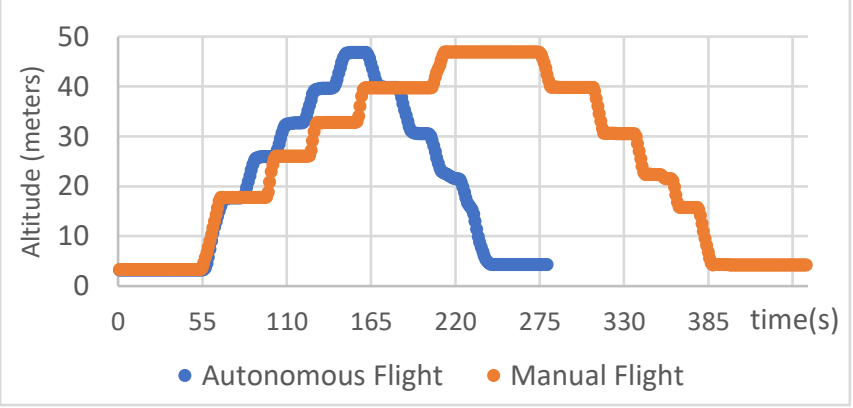

Fig. 7 Visualization of the second test: Altitude

Repeated execution of the trajectory autonomously took on average $45 \%$ less time (Fig. 7) than the user's original flight while maintaining the same rotation of the UAV, camera tilt and quality of the created images.

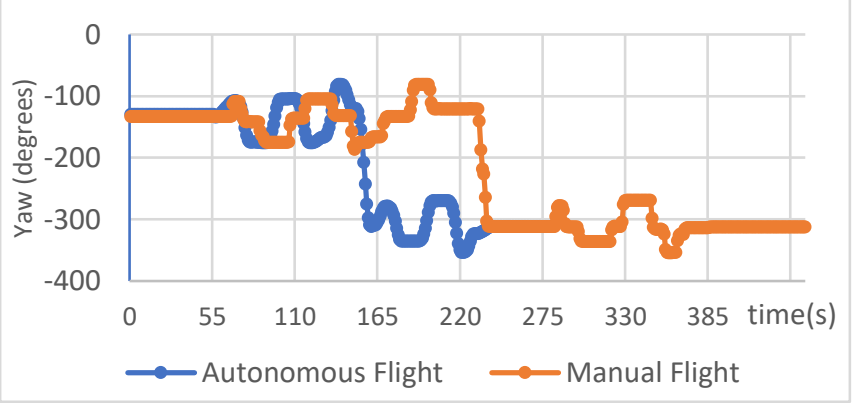

Fig. 8 Visualization of the second test: Yaw 
This significant reduction in time was caused by a continuous change of the UAV's attitude and the camera tilt during the flight between the individual waypoints on the trajectory (Fig. 8). During the manual flight, this operation required stopping and adjusting the appropriate tilt of the camera manually.

The increase in the time efficiency of the flight performance is also visible in Figure 9, which visualizes the development of the GPS coordinates. The autonomous flight minimizes the required time to find an appropriate viewpoint at individual waypoints, to stabilize the UAV, focus, and record creation.

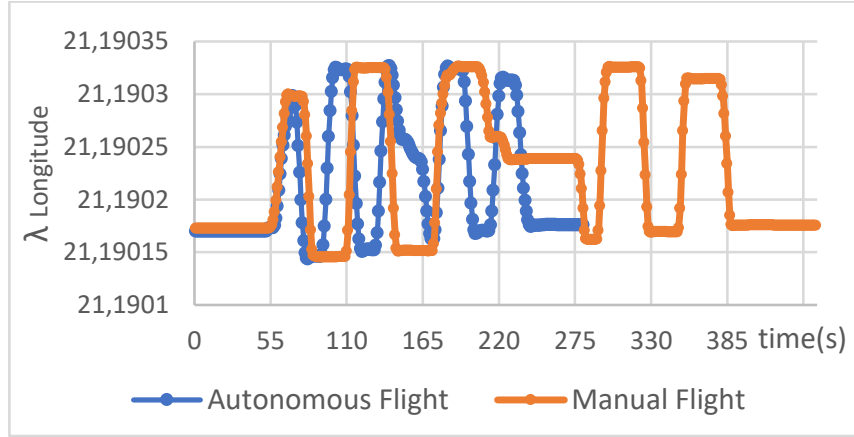

Fig. 9 Visualization of the second test: Longitude

The time efficiency in repeating the flight trajectory is much higher, as no emphasis was placed on the quality and complexity of the inspection process itself when creating the reference trajectory. For a more appropriate demonstration of the developed solution, the reference point for taking the images was the approximate center of the flight trajectory, which enabled the creation of several waypoints with different points of view in a relatively short time. When performing the actual inspection work by a professional, the time required to find an appropriate point of view is much higher and requires a more precise flight with a more extensive number of created images of better quality.

\section{CONCLUSION AND FUTURE WORK}

This paper presents an approach towards a UAV-based flight mission-definition system enabling the configuration and autonomous conduction of flight trajectories. The proposed solution was tested and verified in a simulated environment with two different types of testing, comparing the autonomous flight of the UAV with the manual flight performed by the user.

The efficiency comparison of flight trajectory execution shows that, in general, the performance is good enough for the intended purpose. During testing, the autonomous execution of the trajectory reduced the time required in all cases by almost half while achieving the same output as a usercontrolled manual flight. The 3-dimensional flight trajectory visualization expands the orthographic view used in similar solutions and allows detailed configuration of the flight trajectory from multiple perspectives without limitation. The proposed solution not only extends the possibilities of users in creating complex flight trajectories but also significantly contributes to the higher time efficiency of recurrent flights. These recurrent flights allow less experienced pilots to perform routine work without significant time delays and the potential risk of damaging the UAV. Also, replacing the physical presence of workers in an inaccessible and potentially hazardous environment with machines allows the transfer of the value of highly qualified experts to a safer environment on the ground.

The current state of implementation represents only a part of the final solution and provides several possibilities for expansion in the future. With a web interface, the application can serve as an enterprise solution for managing objects of entire industrial infrastructures with multiple operators and UAVs. Implementing Artificial Intelligence like Machine Learning, Big Data and Computer Vision algorithms can significantly increase the possibilities of automation in postprocessing and routine inspections, thanks to the analysis of the created records and the evaluation of the degradation of scanned objects over time.

\section{ACKNOWLEDGMENT}

The work was supported by The Slovak Research and Development Agency under grant no. APVV-16-0213.

\section{REFERENCES}

[1] Xue, J., \& Su, B. (2017). Significant remote sensing vegetation indices:A review of developments and applications. Journal of Sensors, 2017. https://doi.org/10.1155/2017/1353691

[2] Radoglou-Grammatikis, P., Sarigiannidis, P., Lagkas, T., \& Moscholios, I. (2020). A compilation of UAV applications for precision agriculture. Computer Networks, 172(January), 107148. https://doi.org/10.1016/j.comnet.2020.107148

[3] Arenella, A.; Greco, A.; Saggese, A.; Vento, M., Real Time Fault Detection in Photovoltaic Cells by Cameras on Drones. In Image Analysis and Recognition, Proceedings ofthe 14th International Conference, ICIAR 2017, Montreal, QC, Canada, 5-7 July 2017; Karray, F., Campilho, A., Cheriet, F., Eds.; Springer International Publishing: Cham, Switzerland, 2017; pp. 617-625.

[4] Addabbo, P., Angrisano, A., Bernardi, M. L., Gagliarde, G., Mennella, A., Nisi, M., \& Ullo, S. L. (2018). UAV system for photovoltaic plant inspection. IEEE Aerospace and Electronic Systems Magazine, 33(8), 58-67. https://doi.org/10.1109/MAES.2018.170145

[5] Hallermann, N., \& Morgenthal, G. (2013). Unmanned aerial vehicles (UAV) for the assessment of existing structures. Long Span Bridges and Roofs - Development, Design and Implementation, September. https://doi.org/10.2749/222137813808627172

[6] Stokkeland, M., Klausen, K., \& Johansen, T. A. (2015). Autonomous visual navigation of Unmanned Aerial Vehicle for wind turbine inspection. 2015 International Conference on Unmanned Aircraft Systems, ICUAS 2015, 998-1007. https://doi.org/10.1109/ICUAS.2015 .7152389

[7] Tudevdagva, U., Battseren, B., Hardt, W., Blokzyl, S., \& Lippmann, M. (2017). UAV-based Fully Automated Inspection System for High Voltage Transmission Lines Unmanned Aerial Vehicle-Based Fully Automated Inspection System for High Voltage Transmission Lines. Automation and software engineering , 1(19).

[8] Liu, X., Miao, X., Jiang, H., \& Chen, J. (2020). Data analysis in visual power line inspection: An in-depth review of deep learning for component detection and fault diagnosis. Annual Reviews in Control, 50(June), 253-277. https://doi.org/10.1016/j.arcontrol.2020.09.002 